\title{
Changes in house rules
}

\author{
Nature Neuroscience will now only publish methods online. We have also amended our rules to clarify authors' and \\ co-authors' responsibilities.
}

$\mathbf{R}$ eaders of Nature Neuroscience will have noticed a new section called Online Methods in our articles, technical reports and resources. We are combining our Methods and Supplementary Methods sections and publishing them as a single Online Methods section. The Online Methods remains an integral part of the main paper, and a PDF download of the paper will automatically contain the Online Methods section. Readers of the print version will be directed to our website to access the methods.

A cornerstone of academic research is that the papers are sufficiently detailed to allow for the full assessment and reproduction of the data and the methods that the authors employed to reach their conclusions. A criticism that has been levied against many high-impact journals is that the methods sections are not sufficiently detailed to allow for this careful scrutiny. Most of our readers access our articles online; moving the methods online allows us to display more detailed methods in a cost-effective manner and helps us move to the 'paper of the future'. As in our previous Methods sections, these Online Methods are copyedited and can contain references. Although these references are only included in the online version of the paper, they will be taken into account for impact factor calculations.

Despite this move, however, we will continue to ask that authors keep their methods sections to a limit of 2,000 words. We are aware that readers' time is valuable, and this word limit is sufficient to accommodate the Methods and Supplementary Methods sections that we previously published in a typical Nature Neuroscience paper. We have already found that there may be a few exceptions that require a relaxing of this word limit and will continue to evaluate such papers on a case-by-case basis. The publication requirements that we previously established for methods must still be met (authors must, for example, still include information about institutional committees that approved the experiments and statements on informed consent or animal welfare as necessary).

We have also recently updated our guide to authors (http:// www.nature.com/neuro/authors/index.html) to make the Author Contribution statements mandatory and to make author responsibilities clearer. In the past, at the acceptance of a paper in Nature Neuroscience, we suggested that the corresponding author add a statement detailing the author contributions in the published version of the paper. Most of our papers previously carried such author contribution statements. Recently, the Nature Publishing Group announced that all Naturebranded journals will require such statements to accompany all original research articles.

The purpose of the Author Contribution statement is to give coauthors the credit that they are due. Increasingly, research is carried out by collaborations from different laboratories, and it has become more important to be clear about the relative contributions of each author. There is no prescribed format for these statements, and authors are free to tailor them to their particular needs. Authors can state that all authors contributed to all aspects of the work or that specific members contributed to the design, commented on the manuscript and so forth. We only require that all authors in the study be mentioned in these statements.

Related to this issue, we have also clarified our policies on authorship (http://www.nature.com/authors/editorial_policies/authorship. html). For collaborative studies, we now require that at least one member of each collaboration group, typically the senior author, take responsibility for their group's contribution. Minimally, these responsibilities include ensuring that the original data is preserved and obtainable for re-analysis, ensuring that the data reported are representative of the original data and that image manipulations are in accordance with the journal's guidelines (http://www.nature. com/authors/editorial_policies/image.html). Senior authors are also responsible for ensuring the proper sharing of data, materials, reagents or algorithms presented in the paper. Corresponding authors, although implicitly responsible for the accuracy and integrity of the data, are solely responsible for communication with the journal and in managing communication with the co-authors. They are required to ensure that all the co-authors are aware of the content of the manuscript and the author list. They must inform co-authors of any issues that arise pre- or post-publication and are solely responsible for ensuring the accuracy of the proofs, including ensuring that the names of all of the co-authors are spelled accurately and that their affiliations are correctly listed. We now require all corresponding authors to certify that they are aware of and that they agree with these policies as part of the online submission process.

Our new guidelines will come as no surprise to most authors, who follow these best-practice rules anyway. Would they have prevented some of the cases of misconduct in science? Probably not, but they may have helped co-authors become more aware of (and accountable for) their roles in a paper. For example, an official inquiry into the Jan Hendrik Schön misconduct affair a few years ago, regarding a string of high-profile papers in condensed matter physics with fabricated data, concluded that co-authors on the Schön had broadly met their responsibilities, but that the committee was unable to make specific judgments, as there was no clear consensus on the responsibilities of individual participants in collaborative research (http://publish.aps.org/ reports/lucentrep.pdf). Establishing clear guidelines on responsibilities will hopefully help address some of these issues. We hope you will agree that these guidelines help to clarify the record and increase transparency and accountability in the reporting of scientific data. 